Bramwell L, Harrad S, Abou-Elwafa Abdallah M, Rauert C, Rose M, Fernandes A, Pless-Mulloli T. Predictors of human PBDE body burdens for a UK cohort. Chemosphere 2017, 189, 186-197.

DOI link

https://doi.org/10.1016/i.chemosphere.2017.08.062

ePrints link

http://eprint.ncl.ac.uk/pub details2.aspx?pub id=245217

Date deposited

$16 / 01 / 2018$

Embargo release date

$22 / 08 / 2018$

Copyright

(C) 2017. This manuscript version is made available under the CC-BY-NC-ND 4.0 license

\title{
Licence
}

This work is licensed under a

Creative Commons Attribution-NonCommercial-NoDerivatives 4.0 International licence

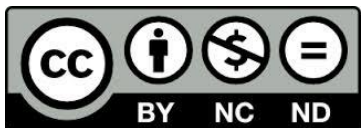




\section{Predictors of human PBDE body burdens for a UK cohort}

Bramwell L ${ }^{1}$

Harrad $S^{2}$

Abdallah M.A.E ${ }^{2}$

Rauert $C^{2}$

Rose M3

Fernandes $\mathrm{A}^{3}$

Pless-Mulloli $\mathrm{T}^{1}$

${ }^{1}$ Newcastle University, Institute of Health and Society, Baddiley-Clark Building, Richardson Road, Newcastle upon Tyne, UK;

${ }^{2}$ Birmingham University, School of Geography, Earth \& Environmental Sciences, Birmingham, UK

${ }^{3}$ Fera Science, Sand Hutton, York, UK; 


\section{Abstract}

$2 \quad$ Human exposure to polybrominated diphenyl ethers (PBDEs) was investigated in a cohort 3 of 20 UK adults along with their anthropometric covariates and relevant properties such as room 4surveys, lifestyle, diet and activity details. Selected PBDE congeners were measured in matched 5 samples of indoor dust, $(n=41)$, vehicles $(n=8)$, duplicate diet $(n=24)$, serum $(n=24)$ and breast 6 milk $(n=6)$.

$7 \quad$ Combined exposure estimates via dust and diet revealed total PBDE intakes of 104 to $81,440 \mathrm{pg} \mathrm{kg}^{-1} \mathrm{bw} \mathrm{d}^{-1}$ for $\sum \mathrm{BDEs}_{3-7}$ and 1,170 to $17,000 \mathrm{pg} \mathrm{kg}^{-1} \mathrm{bw} \mathrm{d}^{-1}$ for BDE-209. These adult 9intakes are well within health reference doses suggested by the European Food Safety Authority 10(EFSA) and the US EPA. For this cohort diet was the primary source of intake of $\mathrm{BDE}_{3-7}$ congeners 11 for the majority of the cohort, with dust the primary source of BDE-209. Primary sources of PBDE 12 exposure vary between countries and regions with differing fire prevention regulations. 13Estimated infant exposures (ages 1.5 to 4.5 years) showed that BDE-99 intake for one of the 14households did not meet EFSA's recommended margin of exposure, a further two households 15were borderline for high level dust and diet intake.

16Males and those having a lower body fat mass had higher serum BDE-153. Higher meat consumption 17 was significantly correlated with higher $\mathrm{BDEs}_{3-7}$ in serum. A reduction in dietary $\mathrm{BDEs}_{3-7}$ would 18therefore result in the greatest reduction in BDE-99 exposure. Rooms containing PUF sofas or 19armchairs over 20 years old had higher BDEs 3 in their dust, and rooms with carpets or rugs of that 20age had higher dust BDE-209. Dusting rooms more frequently resulted in significantly lower 21 concentrations of all major congeners in their dust. Correlation between BDE-209 body burden and 22 dust or diet exposure was limited by its low bioaccessibiliy. Although vehicle dust contained the 23highest concentrations of $\mathrm{BDEs}_{3-7}$ and $\mathrm{BDE}-209$, serum $\mathrm{BDEs}_{3-7}$ correlated most strongly with 24bedroom dust. 


\section{Introduction}

27UK residents are still exposed to a class of potentially harmful brominated flame retardants, 28polybrominated diphenyl ethers (PBDEs), even though European Union regulations 29restricting their manufacture, use and importation came into force in 2004 and 2008. Since 30the 1970s PBDEs have been incorporated into fabrics, foam cushioning and plastics used in 31everyday items such as vehicles, soft furnishings and electronics. PBDEs slow the rate of 32ignition and fire growth in petroleum based polymers and resins. PBDEs are not chemically 33bonded to these materials and are emitted into indoor dust and air through use and 34volatilisation (Rauert and Harrad, 2015; Sjödin et al., 2003). They can then move into the 35wider environment where they have been found in sewage sludge, soils and river and lake 36sediments (Allchin et al., 1999; De Boer et al., 2003; Eljarrat et al., 2008; Harrad et al., 2009). 37They are persistent organic pollutants as defined by the United Nations Environment 38Programme's Stockholm Convention and have an environmental half-life of several years. 39They can travel long distances in the atmosphere and are lipophilic, concentrating in animal 40and marine fats. These qualities and their wide usage have led them to permeate 41environments and food chains around the world (Fromme et al., 2016).

$42 \mathrm{~A}$ recent systematic review of human health consequences of exposure to PBDEs concluded 43health effects may include thyroid disorders, reproductive health effects, and 44neurobehavioral and developmental disorders (Kim et al., 2014). Evidence of these effects 45has been seen in animal and in vitro research, where the mechanism appears to be altered 46hormone regulation (endocrine disruption) (Linares et al., 2015; Marchesini et al., 2008; 47Meerts et al., 2000; Viberg et al., 2006). Exposure during key developmental stages in 48infancy is most damaging as this is the time when altered hormone regulation will have the 49greatest impact. Recent estimates of the economic cost of just the intelligence quotient (IQ) 50points loss and intellectual disability due to PBDE exposure was \$266 billion in the USA and $51 \$ 12.6$ billion in the EU (Attina et al., 2016). These figures must be balanced against amounts 52saved due to fire prevention resulting from furnishing flammability standards e.g. $f 140$ 53million annual savings in the UK estimated by prevention of death, injury and damage to 54property as a result of Furniture and Furnishings Fire Safety Regulations 1988 that require 
55use of flame retardant chemicals. (BIS, 2009). PBDEs were only one group of flame retardant 56chemicals from the several BFR groups commonly used to meet such regulations.

57In 2004, use of two commercial PBDE products, Penta-BDE and Octa-BDE, were restricted 58within the EU (European Council Directive 2003/11/EC) and voluntarily phased out in the 59USA. In 2009, they were added to the Stockholm Convention list of POPs for elimination. 60Penta-BDE had been primarily used in polyurethane foam (PUF) in soft furnishings, vehicles 61 and printed circuit boards, in greatest amounts in the USA. Furnishings could contain one to 62four percent Penta-BDE to comply with fire safety regulations (Hammel et al., 2017). The 63Octa-BDE commercial product has been produced and used less widely than Penta-BDE. Its 64 major use has been in acrylonitrile-butadiene-styrene (ABS) plastics, such as electronics and 65 resin casings of office equipment. The Deca-BDE commercial product has been added to 66furnishing textiles, and in high impact polystyrene (HIPS) for cables, sockets, mobile phones, 67fridges and TV housings.

68Concentrations of BDE-209 are higher in UK indoor dusts than in dusts from mainland 69Europe (Frederiksen et al., 2009; Harrad et al., 2008b) as a result of the UK's more stringent 70fire safety regulations (Furniture and Furnishings Fire Safety Regulations 1988/1989, 1993 71and 2010). Deca-BDE has been restricted from use in electrical and electronic equipment in 72the EU since 2008 and was added to Annex A of the Stockholm Convention list of POPs in 732017. Both diet and contact with indoor dust constitute important exposure pathways for 74PBDEs (Abdallah and Harrad, 2014). Foods from higher up the food chain, of animal origin, 75 with a higher fat content (i.e. fish), meat and dairy have higher PBDE concentrations (EFSA, 762011). PBDEs will be circulating in our food chains for many years to come (Harrad and 77Diamond, 2006), and will be re-circulated back into homes as a result of plastics recycling 78(Samsonek and Puype, 2013) .

79 Whether dust or diet is the primary exposure source for an individual depends on a number 80of factors; loading of PBDE in dust or food items and the amounts ingested, whether and 81 when PBDE technical products have been phased out in that country and on the age of the 82individual (Bramwell et al., 2016a). PBDE intake via ingestion and inhalation of dust is the 83major exposure route for young children in the USA that have frequent hand to mouth 84 behaviours and spend lots of time on floors and carpets (Stapleton et al., 2012). Foetal 85exposure in the womb and transfer of PBDEs from mother to child during breastfeeding are 
86key exposures for children during important developmental periods. For countries outside 87of the US and Canada, the largest contribution to tri-hepta BDE body burden is thought to 88be from diet, especially in regions where Penta-BDE use has been restricted for longer. Dust 89is likely to be most important contributor to exposure to higher brominated congeners in all 90regions (Sahlström et al., 2015).

91The aim of this study was to determine the major dust and diet sources of PBDEs for a north 92east England cohort and to consider any potential health risks. The five specific objectives 93were: (a) to measure PBDE concentrations in dust from homes, work places and vehicles, (b) 94to calculate relative intake of PBDE via dust in the microenvironments, (c) to evaluate the 95relative importance of PBDE exposure via indoor dust versus dietary PBDE exposure, (d) to $96 \mathrm{compare}$ intake estimates with reference health values, (e) to investigate relationships 97between matched environmental and biomonitoring data, and ( $f$ ) to determine the most 98effective means of reducing PBDE exposure for the cohort.

99

\section{Materials and Methods}

101We used a cross sectional and purposive sampling strategy to provide a snap shot of PBDE 102exposures and body burdens for individuals with expected high, average and low exposures. 103By comparing individuals with expected divergent exposures, we aimed to reveal the factors 104influencing body burdens.

\subsection{Volunteer recruitment}

106We targeted individuals with a range of occupations and diets; such as workers in 107electronics, soft furnishings, transport, office workers, outdoor workers, oily fish eaters, 108omnivores and vegetarians. In 2010/11, following ethical approval for the study, volunteers 109over 18 years of age and with six months or more of domestic and occupational stability 110were recruited via local authorities, universities, businesses, hospitals, playgroups and 111 breast-feeding groups. A short pre-screening questionnaire was used to identify volunteers 112that could provide the optimum range of exposures. 79 couples completed the pre113screening questionnaires, 10 couples were invited, and agreed, to participate in the full 
114study week. Further description of the cohort is provided in the Supplementary Information. 115Volunteers gave written informed consent prior to participation.

\subsection{Timing of sample collection}

117Participants undertook a 'sampling week' during which they completed an exposure and 118 food frequency questionnaire (FFQ), food- and activity-diaries, room surveys including 119contents, usage and cleaning information and they were asked not to vacuum or dust their 120home. We adapted the validated WHO-IARC EPIC semi-quantitative dietary questionnaire 121 for the study. On the seventh day of their sampling week, participants collected their 122duplicate diet samples (DD), and the researcher visited that evening to collect the DD 123 samples, home and vehicle dust samples, questionnaires and surveys. The participants then 124fasted until their blood sample collection appointment the following morning where 125 anthropometric measurements were also taken. Two couples repeated the full sampling 126week, with sampling points 6.5 and 7.5 months apart. This provided a longitudinal 127dimension to the study and an element of validation. All sampling weeks took place 128between April $1^{\text {st }} 2011$ and $28^{\text {th }}$ February 2012.

\subsection{Serum, breast milk and duplicate diets}

131Study participants collected an equal amount of whatever food they ate throughout the day 132in a contaminant free (verified by tests carried out prior to sampling) lidded polypropylene 133 container for the 24 hour duplicate diet collection. The next day they provided a fasted 60 $134 \mathrm{ml}$ blood sample at the Clinical Research Facility of the Royal Victoria Infirmary in Newcastle. $13550 \mathrm{ml}$ breastmilk samples were collected by either pump or manual expression up to $12 \mathrm{~h}$ 136before and $24 \mathrm{~h}$ after provision of the blood sample and kept in pre-cleaned Nalgene 137 containers. Samples were stored at $-18^{\circ} \mathrm{C}$ until transfer to the laboratory for analysis. Details 138 of the serum, human milk and duplicate diet sample collection and analysis have been 139published previously (Bramwell et al., 2014; Bramwell et al., 2017).

\subsection{Dust samples}

141Participants were requested not to vacuum or dust their home or vehicle during the 142sampling week. Dust samples from main living areas $(n=11)$, bedrooms $(n=12)$, and vehicles 
$143(n=8)$ were collected by a researcher following a standard sampling protocol to allow direct 144comparison with previous studies (Abdallah and Harrad, 2009; Coakley et al., 2013; Harrad 145et al., 2008a; Harrad et al., 2008b). Samples from workplaces $(n=10)$ were collected during 146the sampling week at the participants' (and their employers') convenience. Dust samples 147were extracted and analysed at the University of Birmingham, UK, using previously 148published methods for preparation, extraction, clean up, analysis and quality control 149(Abdallah et al., 2009; Harrad et al., 2008a; Harrad et al., 2008b). Further details of the dust 150sample collection, preparation, extraction and analysis are provided in the Supplementary 151Information.

\subsection{QA/QC}

153For the analysis of serum, breast milk and duplicate diet samples, the performance 154characteristics of the methodology, including quality assurance parameters such as limits of 155detection (LODs), precision, linear range of measurement, recoveries etc. are included in the 156previous reports (Fernandes et al., 2008; Fernandes, 2004). Further confidence in the data 157is provided by regular and successful participation in laboratory proficiency testing and 158inter-comparison schemes such as POPs in Food 2011 and 2012. PBDEs with IUPAC 159numbers 17, 28, 47, 49, 66, 71, 77, 85, 99, 100, 119, 126, 138, 153, 154, 183 and 209 were 160 measured. The congeners selected for analysis are those for which reference standards are 161available. Typical LODs were 1 to $20 \mathrm{ng} \mathrm{kg}^{-1}$ lipid for PBDEs.

162For the dust sample analysis the average blank (including field blanks) plus 3 standard 163deviations was used for the limit of detection giving an average $0.7 \mathrm{ng} \mathrm{g}^{-1}$ for BDEs-7 (range 1640.2-1.7) and $52 \mathrm{ng} \mathrm{g}^{-1}$ for BDE-209. The PBDE ${ }^{13} \mathrm{C}$ labelled internal standard recoveries were: $165^{13} \mathrm{C}-\mathrm{BDE} 47=69 \pm 20 \%,{ }^{13} \mathrm{C}-\mathrm{BDE} 99=70 \pm 20 \%,{ }^{13} \mathrm{C}-\mathrm{BDE} 153=69 \pm 20 \%$ and ${ }^{13} \mathrm{C}-\mathrm{BDE} 209=17$ $166 \pm 6 \%$. The low recovery for BDE-209 indicates uncertainties in its measurement which are 167presented here with that caveat. Measurement of SRM NIST 2585 had range $78 \%$ (BDE-47) 168to $122 \%$ (BDE-49) and mean $100 \%$ of the certified contents.

\subsection{Exposure Assessment}

170Concentrations of the PBDEs detected in milk and serum samples were lipid-adjusted to 171allow comparison with the literature. PBDE intake for the $24 \mathrm{hrs}$ of the duplicate diet 172collection was measured using whole weight duplicate diet PBDE concentrations multiplied 
173 by the mass of DD collected and divided by the weight of the participant to give $\mathrm{pg} \mathrm{kg}^{-1}$ body 174 weight day ${ }^{-1}$.

175PBDE intakes via dust were estimated by combining measured dust PBDE concentrations 176 with occupation time for individual's various microenvironments (taken from their activity 177diary) using both average ( $20 \mathrm{mg} /$ day) and high ( $50 \mathrm{mg} /$ day) adult dust intake rates average 178and high adult dust ingestion as estimated by Jones-Otazo et al. (2005). Although dust 179ingestion rates may differ between microenvironments and activities (as well as individuals), 180 for the purpose of this study, we have assumed that that dust ingestion occurred pro-rata to 181the proportion of time spent in each microenvironment during the study week. This was 182 considered the only practical approach in the absence of data to confirm any differences 183(Abdallah and Harrad, 2009). For time periods when participants were in their home but 184 not in one of the microenvironments measured, the median of their home dust PBDE 185 concentration was used. For time periods when they were in an indoor environment but not 186in their own home the median of all dusts collected for the study was used. Time spent 187outside was not assigned a PBDE concentration. Intake rates via dust were divided by the 188participant's weight to give pg PBDE intake $\mathrm{kg}^{-1}$ body weight day ${ }^{-1}$.

189PBDE intakes for average and high dust intake scenarios: average $20 \mathrm{mg} \mathrm{d}^{-1}$, high $50 \mathrm{mg} \mathrm{d}^{-1}$ 190(Jones-Otazo et al., 2005) and diet intakes determined from the $24 \mathrm{~h}$ duplicate diet 191concentrations were added together for comparison with the European Food Safety 192Authority's (EFSA) chronic human daily dietary intake estimations to determine the margins 193of exposure (MOEs). As PBDE exposure during infancy is considered to present a greater risk 194to health than that for adults, estimated average and high exposure scenarios for infants 195aged 1.5 to 4.5 years old were developed as well. Daily average $\left(50 \mathrm{mg} \mathrm{d}^{-1}\right)$ and high (200 mg $196 \mathrm{~d}^{-1}$ ) dust intake estimations (Jones-Otazo et al., 2005) per kg body weight were extrapolated 197 from individual adult intake values determined for the study. These were added to average 198and high dietary PBDE intake estimations from the UK total diet study (TDS) (2012) data for 199infants aged 1.5 to 4.5 years old. Risk assessment for infants from PBDE in breast milks 200collected for the study has been previously reported (Bramwell et al., 2014).

\subsection{Data Analysis}


202Associations between PBDE concentrations and intakes and potential predictors were 203explored with scatter plots, box plots and correlations using IBM SPSS Statistics for 204Windows, Version 22.0. Armonk, NY: IBM Corp, Minitab 17 and Excel (Microsoft Office 2052013). The distribution of PBDEs in the different matrices was assessed using Shapiro-Wilk 206statistic. As the majority of distributions were not normal, non-parametric Spearman's 207ranking correlation coefficients were determined. The criteria of $\alpha=0.05$ for statistical 208significance was used. A one sample t test was used to compare PBDE intake of omnivorous 209participants as determined by duplicate diet collection and similar data collected by Harrad 210et al. (2004) to investigate any temporal trend in dietary exposure. Statistical analyses were 211 mostly descriptive and correlations do not have sufficient sample numbers to be robust. 212Details of further statistical analyses of room survey data are presented in the 213Supplementary Information. Where measurements were below limits of detection (LOD) 214 values of LOD*0.5 have been assumed (median bound). $\sum \mathrm{BDEs}_{3-7}$ was calculated as the sum 215of all BDE congeners measured except for BDE-209.

216

\subsection{Human health risk characterisation}

218Potential health risks were calculated from the sum of dust and dietary intake of PBDEs 219using the margin of exposure (MOE) approach as applied by the European Food Safety 220Authority (EFSA) for dietary exposure health risk assessment. The MOE is the ratio of the 221dose at which a small but measureable adverse effect has been reported versus the level of 222exposure of the population under current consideration. The EFSA Panel on Contaminants 223in the food chain (EFSA, 2011) identified effects on neurodevelopment as the critical 224endpoint using BMDL ${ }_{10}$ for neurobehavioural effects in mice induced during a relevant 225period for brain development. Chronic human intakes, associated with body burdens at the 226BMDL ${ }_{10}$ for BDEs-47, $-99,-153$ and -209 , were estimated to be $172,4.2,9.6$ and $1,700,000$ $227 \mathrm{ng} \mathrm{kg}^{-1}$ bw day ${ }^{-1}$ respectively. For PBDEs, EFSA consider that an MOE ratio above 2.5 228indicates that a health concern is unlikely, with risk decreasing as the MOE increases (EFSA, 2292011). It should be noted that although human intakes of concern are presented as daily 230doses these represent chronic intake and as such would be better represented as weekly or 231 monthly intakes as daily intakes can be exceeded on occasion without concern as long as 232other days have lower exposures. 


\section{Results and Discussion}

2350ur cohort consisted of 10 male-female cohabiting couples living in northeast England in 2362011/12. All participants completed full sample and data set collection. Participants were 237recruited from as wide a pool of socio-economic class, occupation, diet and location as 238possible, however, the small number of participants and the focus on breastfeeding 239mothers means that results are not representative of all UK residents' exposures. The 240benefit of the small cohort was that detailed information could be collected for each 241individual allowing the investigation to include almost all contributing factors in PBDE 242exposure known at the time. Further details of occupations, diets, parity, breastfeeding and 243other lifestyle and anthropometric factors are presented in Supplementary Information. 244Previously published serum, breastmilk, and duplicate diet concentrations (Bramwell et al., 2452014; Bramwell et al., 2016b) have been further examined in this investigation, along with 246new matched dust concentrations, diet and dust intake estimations and exposure and food 247 frequency questionnaire, seven day food and activity diary and room survey information in 248order to provide as complete a picture of participants' PBDE exposures as possible.

\subsection{Dust PBDE concentrations}

250Dust samples were collected from 40 micro-environments frequently used by the study 251participants. Main living areas $(n=10)$, bedrooms $(n=12)$ and home offices $(n=2)$ were 252sampled. Workplaces were sampled if access was granted by employers $(n=8)$. None of the 253domestic samples were from open plan homes. Four of the workplace samples were from 254open plan indoor spaces. Vehicles were sampled if participants regularly spent more than 255five hours each week in them $(n=8)$. We measured PBDEs in dust from all of the 256microenvironments sampled. Individual concentrations for all PBDEs in each dust sample 257are presented in Supplementary Information Tables SI 1-4 and summaries of the dust 258 concentrations in different rooms are presented in Table 1. Median dust $\sum \mathrm{BDEs}_{3-7}$ 259concentrations were highest in vehicles $\left(179 \mathrm{ng} \mathrm{g}^{-1}\right)$ followed by living rooms, bedrooms 260then workplaces $\left(137,102\right.$ and $84 \mathrm{ng} \mathrm{g}^{-1}$ respectively). Median BDE-209 concentrations in 261dust were also highest in vehicles $\left(19,000 \mathrm{ng} \mathrm{g}^{-1}\right)$ then bedrooms, living rooms and 262 workplaces $\left(3,530,2,960\right.$, and $2,300 \mathrm{ng} \mathrm{g}^{-1}$ respectively). The highest concentration of 
$263 \sum$ BDEs $_{3-7}$ was measured in a bedroom $\left(7,320 \mathrm{ng} \mathrm{g}^{-1} \mathrm{dust}\right)$, the highest BDE-183 in the rear of 264a work van $\left(367 \mathrm{ng} \mathrm{g}^{-1}\right)$ and the highest BDE-209 in a car $\left(137,000 \mathrm{ng} \mathrm{g}^{-1}\right)$. Summaries of dust 265PBDE concentrations in the different microenvironments are compared with previous UK 266and international data in Table 2. Measurements in this study were in keeping with 267previously published UK data (Harrad et al., 2008a; Harrad et al., 2008b; Pless-Mulloli et al., 2682006; Sjödin et al., 2008) and in agreement with the theory that BDE-209 usage was greater 269in the UK (Fromme et al., 2016; Harrad, 2015). Results were directly comparable to studies 270 by Harrad et al. (2008a; 2008b) as we used the same sampling protocol, sampling 271equipment and laboratory techniques.

272 We compared room survey information such as counts and age of soft furnishings and 273electronics and room cleaning frequencies with the concentrations of PBDEs in each room. 274Details from individual room surveys are provided in Supplementary Information Table SI5. 275We did not find that simple counts of soft furnishings or electronics were good predictors of 276high or low PBDE loading. The clearest association between room contents and PBDE 277concentrations in dust were for BDE-209 if the room contained a carpet or rugs over 20 278years of age (see Supplementary Information Figure 2) . Counts of large PUF items over 20 279years old or office chairs from the USA (adhering to Californian state fire retardancy 280regulations TB117) correlated significantly with concentrations of Penta mix BDEs only, BDE$28147(r=0.37, p=0.036),-99(r=0.35, p=0.047)$ and $\sum B E_{3-7}(r=0.37, p=0.039)$. Higher dusting 282frequency demonstrated the greatest correlation with lower dust PBDE concentrations, with 283BDEs-47, $-99,-153,-154$ and -209 all with correlation significant at the 0.01 level and BDE284100 with correlation significant at the 0.05 level. Table SI 6 in the Supplementary 285 Information contains further correlation data. Discussion of apparent differences between 286repeat sampling weeks' dust data is provided as Supplementary Information.

287 We found that concentrations of $\sum$ Penta product BDEs in the bedroom were significantly 288correlated with those in all other environments measured; living rooms ( $r=0.43, p=0.05)$, 289 workplaces $(r=0.71, p=0.05)$ and vehicles $(r=0.90, p=0.02)$. Concentrations of $\sum$ Penta product 290BDEs in living room dusts correlated strongly with those in workplaces ( $r=0.90, p=0.01)$ but 291not vehicles $(r=0.30, p=0.60)$. A larger data set may have revealed alternative findings, 292particularly for workplaces and vehicles. We suggest that dust particles may briefly adhere 293to and then be shaken from skin, hair, clothing and footwear causing distribution among key 
294environments used by participants. Further correlation data is provided in Supplementary 295Information Table SI13.

\subsection{Intake of PBDEs via dust}

297The ranges of average (20 mg dust ingested $\mathrm{d}^{-1}$ ) and high (50 mg dust ingested $\mathrm{d}^{-1}$ ) PBDE 298intakes via dust for our study participants was $13.8-1,010$ and $35-2,520 \mathrm{pg} \mathrm{kg}^{-1} \mathrm{bw} \mathrm{day}^{-1}$ for $299 \sum \mathrm{BDEs}_{3-7}$, with 281 to 15,900 and 702 to $39,600 \mathrm{pg} \mathrm{kg}^{-1} \mathrm{bw} \mathrm{day}^{-1}$ for BDE-209 via dust. Our $300 \sum \mathrm{BDEs}_{3-7}$ estimates were similar to previous UK and German $\sum \mathrm{BDEs}_{3-7}$ intake estimates 301(Fromme et al., 2009; Harrad et al., 2008a) and an order of magnitude lower than those in 302the USA (Harrad et al., 2008b). In contrast, our BDE-209 intakes from dust were similar to 303those of the USA (Harrad et al., 2008b) and an order of magnitude higher than Belgian and 304German estimates (Fromme et al., 2009; Roosens et al., 2009) (see Supplementary 305 Information Table 6). The wide range of intakes reflected the diverse PBDE loadings 306measured in microenvironment dusts. For this cohort, the influence of specific items in 307specific microenvironments could be reasonably speculated on a case by case basis. 308However, although we expected our participant with occupational PUF and furnishing fabric 309exposure to have a raised PBDE body burden, their fastidious cleaning habits appear to have 310reduced their exposure.

311The greatest proportion of the estimated dust intake for $\sum \mathrm{BDEs}_{3-7}, \mathrm{BDE}-183$ and BDE-209 312 took place in the bedroom (means $43 \%, 38 \%$ and $33 \%$ respectively) due to the greater 313amount of time spent in bedrooms. Workplaces and living rooms were the second most 314important microenvironments for $\sum \mathrm{BDEs}_{3-7}$ exposure (mean 19\%, 13\%) and BDE-183 (20\%, 31521\%). Vehicles were the second most important microenvironment for BDE-209 intake 316(20\%). The relative proportions of PBDE intakes in different microenvironments for 317individual participants is illustrated in Figure 1. Our finding that the greater proportion of 318exposure to all congeners occurs in the bedroom is in keeping with our finding of an 319association between bedroom dust and serum concentrations of the PBDE congeners found 320in the commercial Penta-BDE products (BDE-47, $-99,-100,-153)(r=0.42, p=0.04)$, an 321association that has also been reported elsewhere (Ali et al., 2014; Coakley et al., 2013; 322Watkins et al., 2012). Relationships between PBDE in dust and body burdens 
323We compared PBDE concentrations in dust in the different indoor environments with their 324matched PBDE body burdens. Significant associations were noted between Penta-mix BDEs $325 \mathrm{in}$ bedroom dust and serum $(r=0.45, p=0.04)$. BDE-153 in bedroom dust was significantly 326associated with BDEs-47 ( $r=0.45, p=0.03),-99(r=0.45, p=0.03),-209(r=0.41, p=0.05)$ and $327 \sum \operatorname{BDEs}_{3-7}(r=0.45, p=0.03)$ in serum. BDE-153 in serum was associated but not significantly 328 with BDEs-153 $(0.39,0.06)$ and $\sum \operatorname{BDEs}_{3-7}(0.39,0.06)$ in bedroom dust. BDE-47 was 329associated but not significantly in living room dust and breast milk $(0.77,0.07)$. BDE-209 was 330significantly correlated in serum and workplace dusts $(0.72,0.02)$ however this was strongly 331influenced by one data point. Also correlated but not significantly in workplace dusts were 332BDEs-47 $(0.57,0.07)$ and $-99(0.53,0.09)$. Table SI 7 in Supplementary Information provides 333further dust and body burden correlation data. No significant correlations were found 334between vehicle dust and serum despite vehicles having the highest PBDE concentrations in 335their dust, possibly due to participants spending less time in their cars than in other 336environments measured. The associations between bedroom dust and serum might be 337expected due to participants spending the greatest proportion of their day in this room, 338similarly for associations with workplace dust and serum.

\subsection{Dietary intake of PBDEs}

341We estimated participants' PBDE intake from diet using three different methods, (i) a 24 342 hour duplicate diet sample collected the day before taking serum and milk samples, (ii) a 343seven day food diary completed the seven days prior to serum and milk sampling and (iii) a 344 food frequency questionnaire (FFQ) to represent longer term eating habits. Concentrations 345of PBDEs in the 24 hour duplicate diet samples summarised in Table 1. BDEs-7 were 346 measurable in all of the duplicate diet samples and BDE-209 in 79\% of them. 24 hour 347duplicate diet PBDE concentrations were converted to daily dietary intake estimates which 348ranged from $82-1,320 \mathrm{pg} \mathrm{kg}^{-1}$ bw for $\sum \mathrm{BDEs}_{3-7}$ and $<0.8-1,860 \mathrm{pg} \mathrm{kg}^{-1}$ bw for BDE-209. BDE349209 made up a median of $73 \%$ of the total PBDE exposure from diet. Estimates of 350individuals' PBDE intake via diet are provided in Supplementary Information Table SI 11. The 351 mean intake estimates of BDEs-47, $-99,-100,-153$ and -154 for the omnivores in this study 352 were significantly lower than those measured by Harrad et al. (2004) for duplicate diet 353samples collected in the West Midlands of the UK in 2002 ( $p=0.01)$. The 2002 lower bound 
354mean intakes were within the maximum intakes estimated by this study for BDEs $-47,-100,-$ 355153 and -154 and upper bound intakes for BDEs $-47,-100$, and -154 . These findings indicate 356 a reduction in dietary exposure during the 10 years between the two studies, with the 357greatest reductions being for BDE-99 then BDE-153.

358Meat, fish and dairy portion consumption estimates compared well between the FFQ and 359seven day food diaries. Meat portions consumed per week ranged from none to 14 or 15 360(FFQ and diary respectively), with median 6.3 or 8 portions. Fish and seafood portions 361consumed per week ranged from none to 3.5 (maximum for both FFQ and diary), with 362 median 1.8 or 2 portions. Dairy portions consumed per week ranged from none to 25 or 18 363 (FFQ and diary respectively), with median 8.0 or 8.5 portions. A summary of selected 364information from the FFQ, diary and 24h duplicate diet is presented in Table 3.

365

\subsection{Relationships between PBDE in diet, serum and breastmilk}

367We compared PBDE body burdens with concentrations in the duplicate diet finding a 368significant association for $\sum \mathrm{BDEs}_{3-7}$ in both $(r=0.41, p=0.05)$. Serum samples were collected 369 from fasted participants in order for the serum sample to represent the participants' 370background PBDE body burden without influence from recently consumed food. Breastmilk 371samples were not necessarily collected in a fasted state. The complex relationship between 372historic PBDE deposits in adipose tissue, recent diet, serum and breastmilk is beyond the 373 scope of this paper. We found limited correlation between congeners in serum and 374breastmilk (see Supplementary Information Table SI 8), possibly the result of transfer of 375PBDEs from serum to milk varying between different congeners. Mean serum $/ \mathrm{milk}$ ratios 376generally increased with molecular size and hydrophobicity, e.g. 1.3, 3.1 and 6.0 for BDEs37747, -99 and -209. This pattern was in keeping with findings of a 2012 review of PBDE in 378matched serum and breastmilk samples (Mannetje et al., 2012). BDE-153 in the body 379appears to follow a different pattern with a serum/milk ratio of 0.4 , i.e. more in milk than 380serum. 
$382 \mathrm{We}$ found that the number of meat portions consumed in the week prior to sampling had 383significant positive correlations with BDEs-99 ( $r=0.46, p=0.01)-153(r=0.44, p=0.03)$ and $384 \sum$ BDEs $_{3-7}(r=0.43, p=0.04)$ in serum. Further correlation data between dietary information is 385provided in Supplementary Information Table SI9. The UK FSA 2006 TDS found meat 386products (followed by fish) to contribute most to the PBDE intake of the general UK 387population (EFSA, 2011; FSA, 2006). For participants in this study, meat portions consumed 388exceeded fish portions. Our earlier review of associations between PBDE body burden, dust 389and diet (Bramwell et al., 2016a) also found eating meat to be the most frequently reported 390association (eating dairy and fish were next). Similarly, a nationwide study in the USA found 391 vegetarians to have $23 \%$ lower, and heavy red meat consumers to have $18 \%$ higher total 392PBDEs in serum than omnivores (Fraser et al., 2009).

393

\subsection{Anthropometric and questionnaire covariates of PBDE body burden}

395As well as participants' height, weight and body fat mass measurements, information on 396travel habits, hand to mouth behaviours, parity, numbers of household members, hobbies 397and occupations was also collected to look for indicators of higher serum and breast milk 398PBDE concentrations. These associations are presented in Supplementary Information Table 399SI10. We found serum BDE-153 concentrations to be significantly associated with sex ( $r=-$ 4000.60, $p=0.01)$, percentage of body fat mass $(r=-0.49, p=0.02)$, parity in women $(r=-0.57$, $401 p=0.05)$ and working with electronics $(r=0.59, p=0.01)$. Males generally had higher BDE-153 402in serum than females, in keeping with the findings of a recent Swedish study of 170 adults 403(Bjermo et al., 2017) and a nationwide study in the USA that found males generally had 404higher $\mathrm{BDE}_{3-7}$ body burdens (Fraser et al., 2009). We hypothesise there may be two factors 405influencing the higher serum concentrations of males in this study, (i) men generally had 406lower BMI values; seven of the females had recently been pregnant which would increase 407their BMI and (ii) 9 of the 10 female participants in the study had undergone some 408depuration effect during pregnancy and breast feeding which their male partners had not. In 409a study of the breastmilk of 83 women at three and 12 months postpartum, BDE-153 410showed a significant increase over time (Daniels et al., 2010) suggesting that BDE-153 411present in adipose fat compartments from historic exposures may have been mobilised 412during the nursing period. Storage of BDE-153 in fat compartments in the body has been 
413suggested as the reason for dilution in the serum of people with higher BMI (Cequier et al., 4142015; Fraser et al., 2009). Why these findings for BDE-153 are not consistent with findings 415 for other congeners is not clear but it may be linked to its longer human half-life (Geyer et 416al., 2004).

417

\subsection{Was diet or dust the major source of PBDE exposure for this cohort?}

419Diet was the major source of $\sum \mathrm{BDEs}_{3-7}$ for this cohort making up a median of $85 \%$ of the total 420intake when using duplicate diet data with the average dust ingestion estimate of $20 \mathrm{mg} \mathrm{d}^{-1}$. 421This was a somewhat lower proportion than comparable previous studies estimates of 95\% 422(UK), 96\% (Belgium) and 97\%, (Germany) (Abdallah and Harrad, 2014; Fromme et al., 2009; 423Roosens et al., 2009) due to our higher median $\sum \mathrm{BDEs}_{3-7}$ dust concentration and the notably 424higher concentration of $\sum_{\text {BDEs3-7 }}$ in the German duplicate diets (see Table SI 6). We did not 425include estimates of intake of PBDEs from indoor air in our totals. Previous studies have 426found PBDE intake from air to constitute $<1 \%$ of total PBDE intake (Fromme et al., 2009) and 427a maximum of $2 \%$ (Abdallah and Harrad, 2014).

428Considering only a cohort's average intake hides the substantial variation between 429individuals and their exposure sources - something this study has been able to demonstrate 430clearly (see Figure 2 and Supplementary Information Table SI 6). An individual's total PBDE 431intake is a combination of dust concentrations in different environments, time spent in 432them and dietary habits. For example, the proportion of $\sum \mathrm{BDEs}_{3-7} \mathrm{BDE}$ intake provided by 433dust for an average dust intake rate had a median $4 \%$ but ranged between $0.7 \%(8 \mathrm{M})$ and $43432 \%(5 F)$. Both these participants lived rurally, the former on a smallholding, the other on a 435farm. 8M spent the most time outdoors (almost 9 hours each day), had a low Penta-BDE 436loading in their bedroom dust and, despite a generally home-grown and organic diet, a 437duplicate diet intake in the $3^{\text {rd }}$ quartile. $5 F^{\prime}$ 's relatively high dust intake (32\% using average 438dust intake and 54\% using high dust intake rates) was due to having the room (bedroom) 439 with the highest $\sum \mathrm{BDEs}_{3-7}$ concentrations measured in the study. Although $5 \mathrm{~F}$ consumed a 440vegetarian diet their dietary $\sum \mathrm{BDEs}_{3-7}$ intake was in the top quartile.

441Dust was the greatest source of BDE-209 for our entire cohort, with median intakes making 442up $75 \%$ and $88 \%$ of the total BDE-209 intake for average and high dust intake rates 
443respectively, lower than previous UK estimates of 94\% and 99\% (Abdallah and Harrad, 2014; 444Harrad, 2010) possibly due to declining use of Deca-BDE product and differences between 445 cohorts in the different studies. Individual participants' proportion of total BDE-209 intake 446 provided by dust for average dust intake rate ranged from $14 \%$ ( $8 \mathrm{M}$ ) to $100 \%$ (1Fii and 4471Mii). Participant $10 \mathrm{M}$ had a significantly greater BDE-209 concentration than their partner 448 possibly a reflection of the relatively high amount of time spent in their vehicle $(23 \%$ of their 449time) and BDE-209 concentration in their car (30,338 ng/g).

$450 \mathrm{We}$ found the range of individuals' intakes of $\sum \mathrm{BDEs}_{3-7}$ from dust to be five times greater 451than their intakes from diet. The highest total intake (using average dust intake scenario) 452was 16 times greater than the lowest reported intake. Our data agrees with previous 453hypotheses that the wide range in PBDE concentrations in room dusts (compared with the 454range seen in diets) may be the reason some individuals have significantly higher internal 455dose (Harrad et al., 2008b; Petreas et al., 2003; Thomas et al., 2006; Wu et al., 2007). Dust 456generation, dust ingestion rates, and cleaning frequencies (both microenvironments and 457hand washing) may also be influential.

4580ur study corroborates previous studies findings that average PBDE intakes in the UK are broadly 459similar to those in mainland Europe, where meat is the major source of Penta-BDEs for the average 460person but dust is the major source of BDE-209 (Bramwell et al., 2016a; Harrad et al., 2008b). For 461infants, the average contribution to total intakes from diet were $>90 \%$ for $\sum \mathrm{BDEs}_{3-7}$ and $69 \%$ for $\mathrm{BDE}-$ 462209. At the high dust ingestion rate this decreased to $35-50 \%$ for $\sum \mathrm{BDEs}_{3-7}$ and $88 \%$ for BDE-209. 463These figures indicate similar proportional intake for infants from diet to our adults, although with 464considerably higher amounts ingested per kg body weight (see Table 3).

465

\subsection{Study Limitations}

467This study involved a relatively small cohort of 20 individuals (10 UK couples). The study philosophy 468 concentrated more on the details and habits of the volunteers in order to understand their 469individual exposures. The volume of usage of PBDE mixtures such as PentaBDE, the timelines of 470product introduction and restriction, either voluntary or regulation enforced, and the type of usage, 471are all variables in general population exposure. For example, a far greater volume of the PentaBDE 472mixture was used in the USA and Canada compared to Europe and this is reflected in the relatively 473higher concentrations of related congeners measured in serum, and in house dust levels from North 
474America. Also, where we found diet to be the most important exposure pathway for Penta mix BDEs, 475studies such as (Lorber, 2008) have shown that dust is a major pathway for PentaBDE in North 476American populations. When personal details and habits are considered, the exposure assessment 477is even more unique. Thus, the finding of this study are not intended to be representative of the UK 478as a whole, or even less, other regions of the world.

479

\subsection{Risk characterisation}

481The most relevant congener from a health risk perspective is BDE-99 but there is no 482agreement on a safe intake. The US-EPA suggests a reference dose $100 \mathrm{ng} / \mathrm{kg}$ bw/day (US483EPA, 2006) whereas the more recent EFSA suggested health reference value is $4.2 \mathrm{ng} / \mathrm{kg}$ 484bw/day with an MOE of 2.5 (EFSA, 2011). We investigated potential health risk from our 485estimated PBDE intakes by comparing them with both these reference values (see Table 4 486and Table SI12). The combined uncertainties from household types, sampling and 487 measurement is likely be quite high and should be borne in mind. No health concerns are 488expected from the PBDE intakes estimated in this study for adults as all had MOEs over 2.5 489(EFSA, 2011). The lowest adult MOEs were 2.8 and 3.7 for BDE-99 using a high dust intake 490rate for household 5 with the high $\mathrm{BDE}_{3-7}$ measurements in their bedroom. Accordingly, 491estimated infant daily exposures to BDE-99 for the same home have MOEs below those 492recommended by EFSA for chronic exposure. Using average diet intake data from the 2012 493UK TDS with dust exposure data from this study with average dust intake rates we found the 494lowest MOE estimation to be 2.3 which is similar to the EFSA recommended MOE of 2.5 495deemed to indicate a potential health risk. Using high dust intake rates with dust data for 496this study and $97.5^{\text {th }}$ percentile (P97.5) dietary intake estimates from the 2012 UK TDS this 497MOE dropped to 0.7 and two additional homes indicated high infant intake MOEs between 4982.5 and 3. All other adult and infant MOEs using EFSA reference values and all MOEs using 499US EPA values were comfortably above the recommended MOE. Follow-up measurement of 500the PBDE body burdens for infants of parents participating in this study could help describe 501associations with raised intake estimations. 
504This detailed study is the first anywhere to document concentrations of PBDEs, including 505BDE-209, in samples of indoor dust and diet with matched human serum and breast milk 506 concentrations. Our findings confirmed that both diet and dust make a contribution to PBDE 507 body burdens and provide new evidence of a wide range in their relative contributions 508between individuals. Diet appeared to be the primary source of intake of $\mathrm{BDE}_{3-7}$ congeners 509 for the majority of this cohort, and meat consumption demonstrated the strongest 510significant positive association between diet type and serum BDEs3-7 concentrations. Dust 511 was the cohort's primary source of BDE-209. Rooms containing a carpet or rugs over 20 512 years old had higher BDE-209 concentrations in their dust. Rooms that were dusted more 513frequently had less BDE-209, as well as less Penta mix PBDE congeners. Rooms containing 514sofas or armchairs over 20 years old had higher concentrations of commercial Penta mix 515PBDE congeners. BDE-209 concentrations in room dusts did not widely correlate with BDE516209 body burdens, possibly due to the congener's relatively large molecular size and low 517bioaccessibility. Correlations between $\mathrm{BDE}_{3-7}$ congeners in serum and indoor dust were 518strongest in bedrooms in keeping with the greater proportion of time spent there. Being 519male and having a lower body fat mass were indicators of higher serum BDE-153 for this 520cohort. BDE-99 was the congener demonstrating the lowest MOE (and therefore the 521greatest health risk) and although we found a reduction in dietary exposure to this and 522other Penta-mix PBDEs since 2002, reducing dietary exposure would still have the greatest 523 effect in reducing body burdens.

\section{Acknowledgements}

525This study was funded by the UK Natural Environment Research Council (NERC): CASE 526studentship NE/F014139/1, the Institute of Health and Society, Newcastle University, UK, 527and Fera, Sand Hutton, York, UK. Thanks are extended to Dr David Mortimer, FSA, London, 528UK for expertise and advice regarding furniture fire safety regulations and dietary exposure 529to PBDEs. Thanks are also given to the study participants. 


\section{References}

534Directive 2003/11/EC of the European Parliament and of the Council of 6 February 2003 amending 535 for the 24th time Council Directive 76/769/EEC relating to restrictions on the marketing and use of 536 certain dangerous substances and preparations (pentabromodiphenyl ether, octabromodiphenyl 537ether) L42/45 J 2003.

538The Stockholm Convention on Persitant Organic Pollutants available at http://chm.pops.int/ 539accessed 13.10.16.

540(DCA), S.o.C.D.o.C.A., 2013. Technical Bulletin 117-2013 Requirements, Test Procedure and 541Apparatus for Testing the Smolder Resistance of Materials Used in Upholstered Furniture viewed 54217.11.16 http://www.bearhfti.ca.gov/about_us/tb117_2013.pdf.

543Abdallah, M.A., Harrad, S., 2009. Personal exposure to HBCDs and its degradation products via 544ingestion of indoor dust. Environ Int 35, 870-876.

545Abdallah, M.A., Harrad, S., 2014. Polybrominated diphenyl ethers in UK human milk: implications for 546infant exposure and relationship to external exposure. Environ. Int. 63, 130-136.

547Abdallah, M.A., Harrad, S., Covaci, A., 2009. Isotope dilution method for determination of 548 polybrominated diphenyl ethers using liquid chromatography coupled to negative ionization 549atmospheric pressure photoionization tandem mass spectrometry: validation and application to 550house dust. Anal Chem 81, 7460-7467.

551Ali, N., Mehdi, T., Malik, R.N., Eqani, S.A.M.A.S., Kamal, A., Dirtu, A.C., Neels, H., Covaci, A., 2014. 552Levels and profile of several classes of organic contaminants in matched indoor dust and serum 553samples from occupational settings of Pakistan. Environ. Pollut. 193, 269-276.

554Allchin, C.R., Law, R.J., Morris, S., 1999. Polybrominated diphenylethers in sediments and biota 555downstream of potential sources in the UK. Environ. Pollut. 105, 197-207.

556Attina, T.M., Hauser, R., Sathyanarayana, S., Hunt, P.A., Bourguignon, J.-P., Myers, J.P., DiGangi, J., 557Zoeller, R.T., Trasande, L., 2016. Exposure to endocrine-disrupting chemicals in the USA: a 558population-based disease burden and cost analysis. The Lancet Diabetes \& Endocrinology.

559Batterman, S.A., Chernyak, S., Jia, C., Godwin, C., Charles, S., 2009. Concentrations and Emissions of 560Polybrominated Diphenyl Ethers from U.S. Houses and Garages. Environ. Sci. Technol. 43, 26935612700 .

562BIS, 2009. A statistical report to investigate the effectiveness of the Furniture and Furnishings (Fire) 563(Safety) Regulations 1988. Department for Business, Innovation and Skills.

564Bjermo, H., Aune, M., Cantillana, T., Glynn, A., Lind, P.M., Ridefelt, P., Darnerud, P.O., 2017. Serum 565 levels of brominated flame retardants (BFRs: PBDE, HBCD) and influence of dietary factors in a 566population-based study on Swedish adults. Chemosphere 167, 485-491.

567Bramwell, L., Fernandes, A., Rose, M., Harrad, S., Pless-Mulloli, T., 2014. PBDEs and PBBs in human 568serum and breast milk from cohabiting UK couples. Chemosphere 116, 67-74.

569Bramwell, L., Glinianaia, S.V., Rankin, J., Rose, M., Fernandes, A., Harrad, S., Pless-Mulolli, T., 2016. 570Associations between human exposure to polybrominated diphenyl ether flame retardants via diet 571and indoor dust, and internal dose: A systematic review. Environ. Int. 92-93, 680-694.

572Bramwell, L., Mortimer, D., Rose, M., Fernandes, A., Harrad, S., Pless-Mulloli, T., 2017. UK dietary 573exposure to PCDD/Fs, PCBs, PBDD/Fs, PBBs and PBDEs: comparison of results from 24-h duplicate 574diets and total diet studies. Food Additives \& Contaminants: Part A 34, 65-77. 
575Cequier, E., lonas, A.C., Covaci, A., Marcé, R.M., Becher, G., Thomsen, C., 2014. Occurrence of a 576Broad Range of Legacy and Emerging Flame Retardants in Indoor Environments in Norway. Environ. 577Sci. Technol. 48, 6827-6835.

578Cequier, E., Marcé, R.M., Becher, G., Thomsen, C., 2015. Comparing human exposure to emerging 579and legacy flame retardants from the indoor environment and diet with concentrations measured in 580serum. Environ. Int. 74, 54-59.

581Civan, M.Y., Kara, U.M., 2016. Risk assessment of PBDEs and PAHs in house dust in Kocaeli, Turkey: 582levels and sources. Environmental Science and Pollution Research 23, 23369-23384.

583Coakley, J.D., Harrad, S.J., Goosey, E., Ali, N., Dirtu, A.C., Van den Eede, N., Covaci, A., Douwes, J., 584Mannetje, A., 2013. Concentrations of polybrominated diphenyl ethers in matched samples of 585indoor dust and breast milk in New Zealand. Environ. Int. 59, 255-261.

586Daniels, J.L., Pan, I.J., Jones, R., Anderson, S., Patterson Jr, D.G., Needham, L.L., Sjödin, A., 2010. 587Individual characteristics associated with PBDE levels in U.S. human milk samples. Environ. Health. 588Persp. 118, 155-160.

589De Boer, J., Wester, P.G., Van Der Horst, A., Leonards, P.E.G., 2003. Polybrominated diphenyl ethers 590in influents, suspended particulate matter, sediments, sewage treatment plant and effluents and 591biota from the Netherlands. Environ. Pollut. 122, 63-74.

592EFSA, 2011. Panel on Contaminants in the Food Chain (CONTAM): Scientific Opinion on 593Polybrominated Diphenyl Ethers (PBDEs) in Food. EFSA Journal 9, 2156-2430.

594Eljarrat, E., Marsh, G., Labandeira, A., Barceló, D., 2008. Effect of sewage sludges contaminated with 595polybrominated diphenylethers on agricultural soils. Chemosphere 71, 1079-1086.

596Fernandes, A., Dicks, P., Mortimer, D., Gem, M., Smith, F., Driffield, M., White, S., Rose, M., 2008. 597Brominated and chlorinated dioxins, PCBs and brominated flame retardants in Scottish shellfish: 598Methodology, occurrence and human dietary exposure. Mol. Nut. Food Res. 52, 238-249.

599Fernandes, A., White, S., D'Silva, K., Rose, M., 2004. Simultaneous determination of PCDDs, PCDFs, 600PCBs and PBDEs in food. Talanta 63, 1147-1155.

601Fraser, A.J., Webster, T.F., McClean, M.D., 2009. Diet contributes significantly to the body burden of 602PBDEs in the general U.S. population. Environ. Health. Persp. 117, 1520-1525.

603Frederiksen, M., Vorkamp, K., Thomsen, M., Knudsen, L.E., 2009. Human internal and external 604exposure to PBDEs - A review of levels and sources. Int. J. Hyg. Envir. Heal. 212, 109-134.

605Fromme, H., Becher, G., Hilger, B., Völkel, W., 2016. Brominated flame retardants - Exposure and 606risk assessment for the general population. Int. J. Hyg. Envir. Heal. 219, 1-23.

607Fromme, H., Korner, W., Shahin, N., Wanner, A., Albrecht, M., Boehmer, S., Parlar, H., Mayer, R., 608 Liebl, B., Bolte, G., 2009. Human exposure to polybrominated diphenyl ethers (PBDE), as evidenced 609 by data from a duplicate diet study, indoor air, house dust, and biomonitoring in Germany. Environ. 610Int. 35 (8), 1125-1135.

611FSA, 2006. Food Surveillance Information Sheet 10/06. in: Agency, F.S. (Ed.). Food Standards Agency.

612Geyer, H.J., Schramm, K.-W., Darnerud, P.O., Aune, M., Feicht, E.A., Fried, K.W., 2004. Terminal 613 elimination half-lives of the brominated flame retardants TBBPA, HBCD, and lower brominated 614PBDEs in humans. Organohalogen Compd. 66, 3867-3872. 
615Hammel, S.C., Hoffman, K., Lorenzo, A.M., Chen, A., Phillips, A.L., Butt, C.M., Sosa, J.A., Webster, T.F., 616Stapleton, H.M., 2017. Associations between flame retardant applications in furniture foam, house 617dust levels, and residents' serum levels. Environ. Int. 107, 181-189.

618 Harrad, S., 2010. The contamination of indoor environments with persistant organic pollutants. in: 619 Harrad, S. (Ed.). Persistant Organic Pollutants. Blackwell Publishing Ltd, Chippenham, Wiltshire, UK.

$620 \mathrm{Harrad}, \mathrm{S} ., 2015$. A meta-analysis of recent data on UK environmental levels of POP-BFRs in an 621 international context: Temporal trends and an environmental budget. Emerging Contaminants 1, 3962253.

623 Harrad, S., Abdallah, M.A., 2011. Brominated flame retardants in dust from UK cars--within-vehicle 624spatial variability, evidence for degradation and exposure implications. Chemosphere 82, 1240-1245.

625 Harrad, S., Abdallah, M.A., Rose, N.L., Turner, S.D., Davidson, T.A., 2009. Current-use brominated 626flame retardants in water, sediment, and fish from English lakes. Environ Sci Technol 43, 9077-9083.

627Harrad, S., Diamond, M., 2006. New Directions: Exposure to polybrominated diphenyl ethers (PBDEs) 628and polychlorinated biphenyls (PCBs): Current and future scenarios. Atmos. Environ. 40, 1187-1188.

629 Harrad, S., Goosey, E., Desborough, J., Abdallah, M.A.-E., Roosens, L., Covaci, A., 2010. Dust from 630U.K. Primary School Classrooms and Daycare Centers: The Significance of Dust As a Pathway of 631Exposure of Young U.K. Children to Brominated Flame Retardants and Polychlorinated Biphenyls. 632Environ. Sci. Technol. 44, 4198-4202.

633Harrad, S., Ibarra, C., Abdallah, M.A.-E., Boon, R., Neels, H., Covaci, A., 2008a. Concentrations of 634 brominated flame retardants in dust from United Kingdom cars, homes, and offices: Causes of 635variability and implications for human exposure. Environ. Int. 34, 1170-1175.

636Harrad, S., Ibarra, C., Diamond, M., Melymuk, L., Robson, M., Douwes, J., Roosens, L., Dirtu, A.C., 637Covaci, A., 2008b. Polybrominated diphenyl ethers in domestic indoor dust from Canada, New 638Zealand, United Kingdom and United States. Environ. Int. 34, 232-238.

639Jones-Otazo, H.A., Clarke, J.P., Diamond, M.L., Archbold, J.A., Ferguson, G., Harner, T., Richardson, 640G.M., Ryan, J.J., Wilford, B., 2005. Is house dust the missing exposure pathway for PBDEs? An 641analysis of the urban fate and human exposure to PBDEs. Environ. Sci. Technol. 39, 5121-5130.

642Kim, S.-K., Kim, K.-S., Sang, H.H., 2016. Overview on relative importance of house dust ingestion in 643human exposure to polybrominated diphenyl ethers (PBDEs): International comparison and Korea as 644a case. Sci. Total Environ. 571, 82-91.

645Kim, Y.R., Harden, F.A., Toms, L.-M.L., Norman, R.E., 2014. Health consequences of exposure to 646brominated flame retardants: A systematic review. Chemosphere 106, 1-19.

647Korcz, W., Struciński, P., Góralczyk, K., Hernik, A., Łyczewska, M., Matuszak, M., Czaja, K., Minorczyk, 648M., Ludwicki, J.K., 2017. Levels of polybrominated diphenyl ethers in house dust in Central Poland. 649Indoor Air 27, 128-135.

650Linares, V., Bellés, M., Domingo, J., 2015. Human exposure to PBDE and critical evaluation of health 651hazards. Arch Toxicol 89, 335-356.

652Lorber, M., 2008. Exposure of Americans to polybrominated diphenyl ethers. J. Expo. Sci. Environ. 653Epidemiol. 18, 2-19.

654Mannetje, A., Coakley, J., Mueller, J.F., Harden, F., Toms, L.M., Douwes, J., 2012. Partitioning of 655 persistent organic pollutants (POPs) between human serum and breast milk: a literature review. 656Chemosphere 89, 911-918. 
657Marchesini, G.R., Meimaridou, A., Haasnoot, W., Meulenberg, E., Albertus, F., Mizuguchi, M., 658Takeuchi, M., Irth, H., Murk, A.J., 2008. Biosensor discovery of thyroxine transport disrupting 659chemicals. Toxicology and Applied Pharmacology 232, 150-160.

660Meerts, I.A.T.M., van Zanden, J.J., Luijks, E.A.C., van Leeuwen-Bol, I., Marsh, G., Jakobsson, E., 661Bergman, Å., Brouwer, A., 2000. Potent Competitive Interactions of Some Brominated Flame 662Retardants and Related Compounds with Human Transthyretin in Vitro. Toxicological Sciences 56, 66395-104.

664Newton, S., Sellström, U., de Wit, C.A., 2015. Emerging Flame Retardants, PBDEs, and HBCDDs in 665Indoor and Outdoor Media in Stockholm, Sweden. Environ. Sci. Technol. 49, 2912-2920.

666Petreas, M., She, J., Brown, F.R., Winkler, J., Windham, G., Rogers, E., Zhao, G., Bhatia, R., Charles, 667M.J., 2003. High body burdens of 2,2',4,4'-tetrabromodiphenyl ether (BDE-47) in California women. 668Environ. Health. Persp. 111, 1175-1179.

669Pless-Mulloli T, S.A., Schilling B, Paepke O, 2006. Levels of PBDE in household dust and lint in the UK, 670Germany and the USA 2006. Organohalogen Compounds 68, 495-498.

671Rauert, C., Harrad, S., 2015. Mass transfer of PBDEs from plastic TV casing to indoor dust via three 672migration pathways - A test chamber investigation. Sci. Total Environ. 536, 568-574.

673Roosens, L., Abdallah, M.A., Harrad, S., Neels, H., Covaci, A., 2009. Factors influencing concentrations 674of polybrominated diphenyl ethers (PBDEs) in students from Antwerp, Belgium. Environ Sci Technol $67543,3535-3541$.

676Sahlström, L.M.O., Sellström, U., de Wit, C.A., Lignell, S., Darnerud, P.O., 2015. Feasibility Study of 677 Feces for Noninvasive Biomonitoring of Brominated Flame Retardants in Toddlers. Environ. Sci. 678Technol. 49, 606-615.

679Samsonek, J., Puype, F., 2013. Occurrence of brominated flame retardants in black thermo cups and 680 selected kitchen utensils purchased on the European market. Food additives \& contaminants. Part A, 681Chemistry, analysis, control, exposure \& risk assessment 30, 1976-1986.

682Santillo, D., Labunska, I., , Davidson, H., Johnston, P., Strutt, M., , Knowles, O., 2003. Consuming 683 Chemicals - Hazardous Chemicals in House Dust as an Indicator of Chemical Exposure in the Home 684Greenpeace Research Laboratories.

685Sjödin, A., Päpke, O., McGahee, E., Focant, J.-F., Jones, R.S., Pless-Mulloli, T., Toms, L.-M.L., 686 Herrmann, T., Müller, J., Needham, L.L., Patterson Jr, D.G., 2008. Concentration of polybrominated 687diphenyl ethers (PBDEs) in household dust from various countries. Chemosphere 73, S131-S136.

688Sjödin, A., Patterson Jr, D.G., Bergman, Å., 2003. A review on human exposure to brominated flame 689retardants - Particularly polybrominated diphenyl ethers. Environ. Int. 29, 829-839.

690Stapleton, H.M., Eagle, S., Sjodin, A., Webster, T.F., 2012. Serum PBDEs in a North Carolina toddler 691cohort: associations with handwipes, house dust, and socioeconomic variables. Environ. Health. 692Persp. 120, 1049-1054.

693Stapleton, H.M., Misenheimer, J., Hoffman, K., Webster, T.F., 2014. Flame retardant associations 694between children's handwipes and house dust. Chemosphere 116, 54-60.

695Thomas, G.O., Wilkinson, M., Hodson, S., Jones, K.C., 2006. Organohalogen chemicals in human 696blood from the United Kingdom. Environ. Pollut. 141, 30-41.

697US-EPA, 2006. Toxicological review of 2,2', 4,4', 5-Pentabromodiphenyl ether (BDE-99). United States 698Environmental Protection Agency, US Environmental Protection Agency, Washington, DC available 699at: www.epa.gov/iris. 
700Viberg, H., Johansson, N., Fredriksson, A., Eriksson, J., Marsh, G., Eriksson, P., 2006. Neonatal 701Exposure to Higher Brominated Diphenyl Ethers, Hepta-, Octa-, or Nonabromodiphenyl Ether, 702 Impairs Spontaneous Behavior and Learning and Memory Functions of Adult Mice. Toxicological 703Sciences 92, 211-218.

704Watkins, D.J., McClean, M.D., Fraser, A.J., Weinberg, J., Stapleton, H.M., Sjodin, A., Webster, T.F., 7052012. Impact of dust from multiple microenvironments and diet on PentaBDE body burden. Environ. 706Sci. Technol. 46, 1192-1200.

707Webster, L., Russell, M., Walsham, P., Moffat, C.F., 2006. Fisheries Research Services Internal Report 708No 06/06: A review of brominated flame retardants (BFRs) in the aquatic environment and the 709development of an analytical technique for their analysis in environmental samples.

710Whitehead, T.P., Crispo Smith, S., Park, J.-S., Petreas, M.X., Rappaport, S.M., Metayer, C., 2015. 711Concentrations of persistent organic pollutants in California women's serum and residential dust. 712Environ Res 136, 57-66.

713Wu, N., Herrmann, T., Paepke, O., Tickner, J., Hale, R., Harvey, E., La Guardia, M., McClean, M.D., 714Webster, T.F., 2007. Human Exposure to PBDEs: Associations of PBDE Body Burdens with Food 715Consumption and House Dust Concentrations. Environ. Sci. Technol. 41, 1584-1589. 